\title{
STEM Investigations of Defects and Interfaces In Complex Oxides
}

\author{
S. J. Pennycook ${ }^{1}$, M. Varela ${ }^{1}$, J. Santamaria ${ }^{2}$, D. Kumar ${ }^{3}$ and G. Duscher ${ }^{1,4}$,
}

${ }^{1}$ Solid State Division, Oak Ridge National Laboratory, Oak Ridge, Tennessee.

${ }^{2}$ Departamento de Fisica Aplicada III, Universidad Complutense de Madrid, 28040 Madrid. Spain

${ }^{3}$ Center for Advanced Materials \& Smart Structures, North Carolina A \& T State University, Greensboro, NC

${ }^{4}$ Department of Materials Science and Engineering, North Carolina State University, Raleigh, NC

The properties of complex oxides can be significantly affected or even dominated by the presence of extended defects or epitaxial strain. Evidence is accumulating that many effects may have a common origin due to non-stoichiometry in the highly stressed region of the dislocation cores. The combination of Z-contrast imaging, electron energy loss spectroscopy (EELS) and first-principles theory is a powerful means to unravel such phenomena. A good illustration is the prototypical perovskite $\mathrm{SrTiO}_{3} \cdot[1]$ Z-contrast images revealed the location of the atomic columns, which provided starting models for theory. Calculations then revealed that the low energy structures that agreed with the images were non-stoichiometric, having a Ti/O ratio greater than the bulk. This in turn was confirmed by atomic-resolution EELS. The theory also showed that the excess d-electrons on the Ti atoms were in the conduction band (which is formed from Ti d orbitals). In an acceptordoped bulk, these electrons would move off the boundary to neutralize nearby acceptors, leaving the boundary charged and setting up a space charge depletion layer. This is the origin of the electrical activity used for varistors, capacitors and other devices.

Dislocations in other complex oxides have similar core structures and are also expected to have strong effects on properties. Band bending due to non-stoichiometric grain boundaries in YBCO leads to hole depletion, and explains the exponential reduction in critical currents with misorientation[2]. In the manganites, grain boundaries have been linked to a useful low-field colossal magnetoresistance. Figure 2 shows the determination of interface structure and misfit dislocation core structure at the $\mathrm{LaAlO}_{3} / \mathrm{La}_{2 / 3} \mathrm{Ca}_{1 / 3} \mathrm{MnO}_{3}$ interface. The interfacial plane can be determined directly from intensity traces across the interface on the two cation sublattices, and the misfit dislocation core is seen to be located two unit cells into the film.

Epitaxial strain may also have a large effect on properties. Figure 3 shows a Z-contrast image of a $\left[\mathrm{YBCO}_{1} / \mathrm{PBCO}_{5}\right]$ superlattice with an EELS spectrum taken from the center of the single YBCO unit cell layer. The pre-edge peak is absent, indicating strong hole depletion. For comparison, the EELS spectrum from the center of the $\mathrm{YBCO}$ layer in a $\left[\mathrm{YBCO}_{8} / \mathrm{PBCO}_{5}\right]$ superlattice does show a substantial pre-edge feature. The reason for the difference is strain; the single layer superlattice is strained, the thicker layer is relaxed. Strain affects the charge in the $\mathrm{CuO}_{2}$ planes and therefore Tc.

\section{References}

[1] .M. Kim et al., Phys. Rev. Letts. 86 (2001) 4056

[2] N D Browning et al Physica C 294 (1998) 183, also S. J. Pennycook et al., Mat. Res. Soc. Symp. Proc. 654 (2001) AA1.3.2

[3] This work was supported by the USDOE under contract DE-AC05-00OR22725 managed by UTBattelle, LLC. 

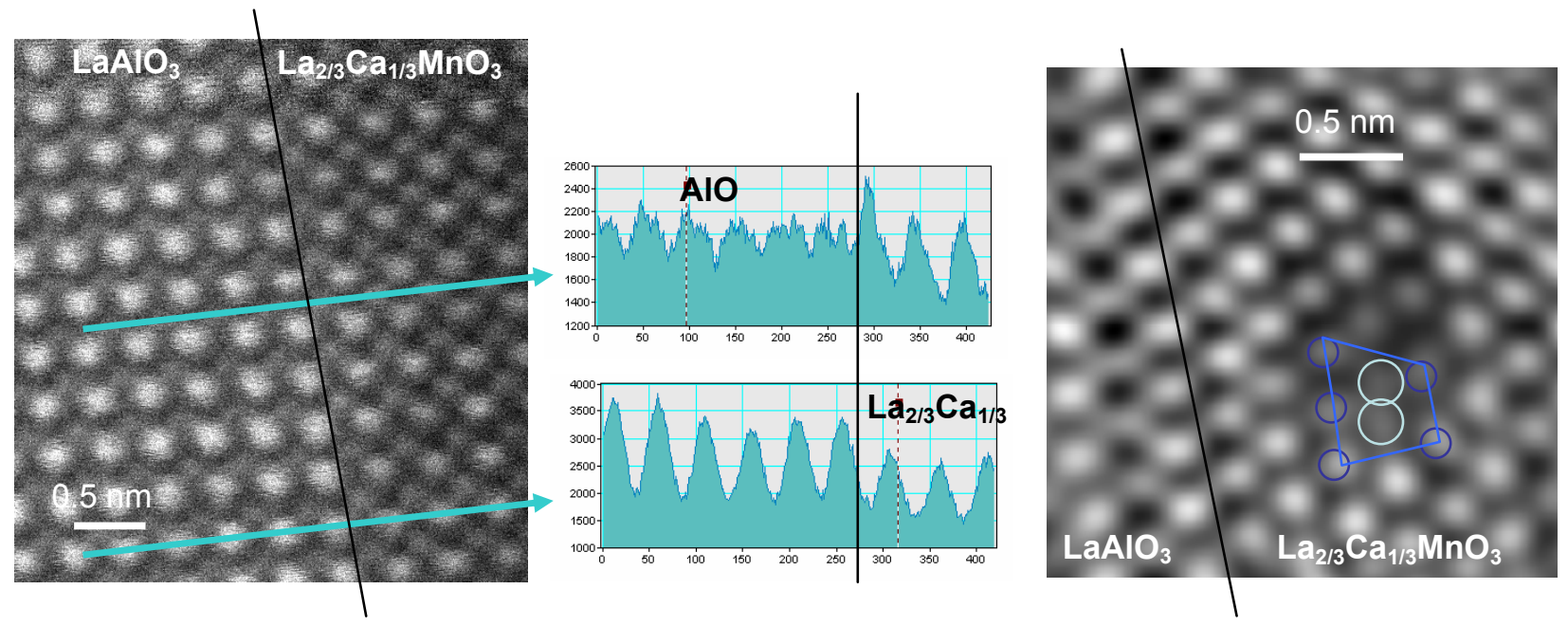

Fig. 1. (a) Z-contrast image of a $\mathrm{LaAlO}_{3} / \mathrm{La}_{2 / 3} \mathrm{Ca}_{1 / 3} \mathrm{MnO}_{3}$ interface showing the location of the interface from intensity traces across the two cation sublattices. (b) Higher magnification filtered image showing the location and core structure of a misfit dislocation.
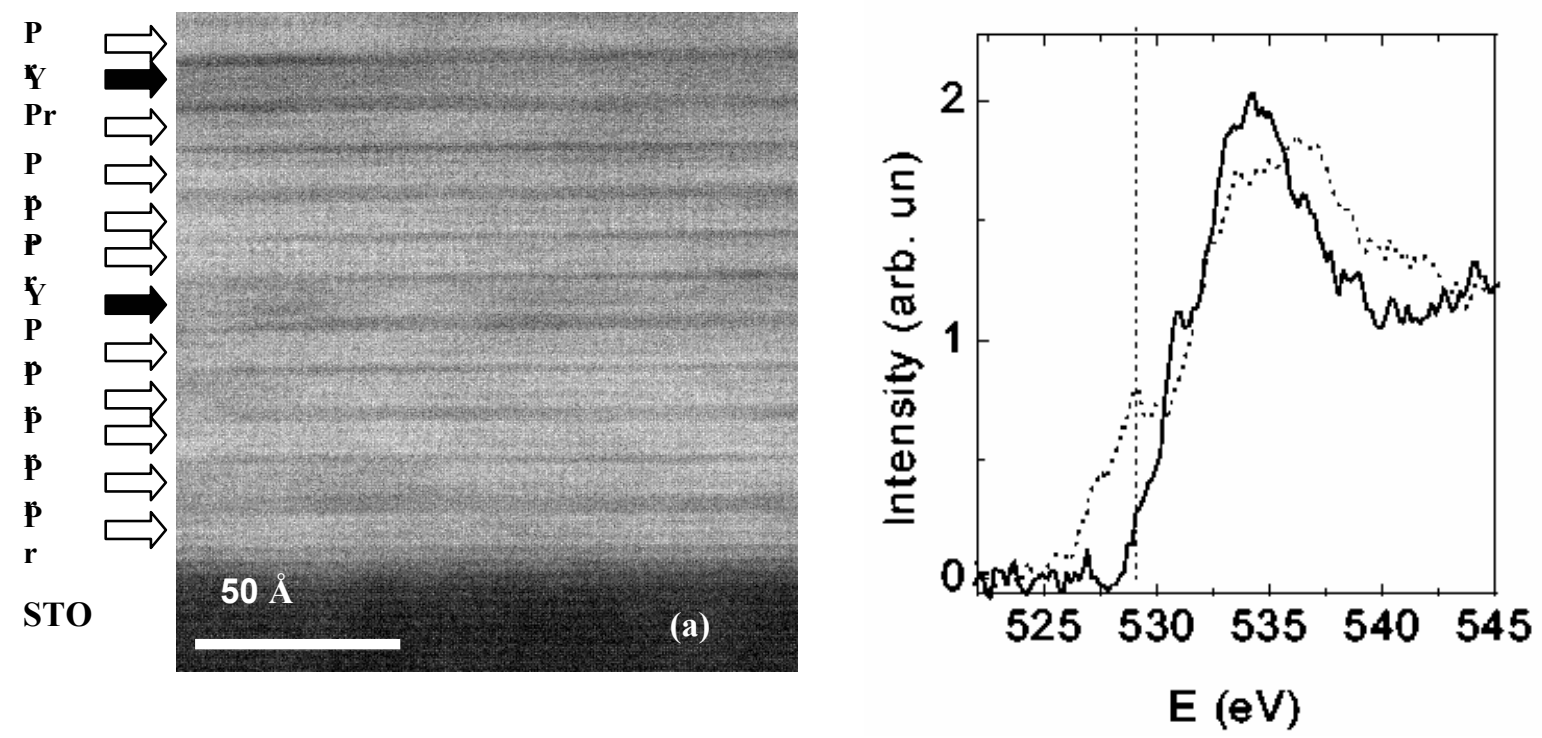

Fig. 2. Z-contrast image of a $\left[\mathrm{YBCO}_{1} / \mathrm{PBCO}_{5}\right]$ superlattice. The EELS spectrum of the $\mathrm{O} \mathrm{K}$ edge shows no pre-peak (solid line), indicating strong hole depletion consistent with the measured Tc of $35 \mathrm{~K}$. A $\left[\mathrm{YBCO}_{8} / \mathrm{PBCO}_{5}\right]$ superlattice (dotted line) does show a pre-peak and a Tc of $88 \mathrm{~K}$. 